\title{
Evidence for low dimensional chaos in sunspot cycles
}

\author{
C. Letellier ${ }^{1}$, L. A. Aguirre ${ }^{2}$, J. Maquet ${ }^{1}$, and R. Gilmore ${ }^{3}$ \\ 1 CORIA/CNRS UMR 6614, Université et INSA de Rouen, Av. de l'Université, BP 12, 76801 Saint-Etienne du Rouvray Cedex, France \\ e-mail: Christophe.Letellier@coria.fr \\ 2 Departamento de Engenharia Eletrônica, Universidade Federal de Minas Gerais, Av. Antônio Carlos 6627, 31.270-901 Belo Horizonte, MG., \\ Brazil \\ 3 Physics Department, Drexel University, Philadelphia, PA 19104, USA
}

Received 29 July 2005 / Accepted 25 November 2005

\section{ABSTRACT}

Sunspot cycles are widely used for investigating solar activity. In 1953 Bracewell argued that it is sometimes desirable to introduce the inversion of the magnetic field polarity, and that can be done with a sign change at the beginning of each cycle. It will be shown in this paper that, for topological reasons, this so-called Bracewell index is inappropriate and that the symmetry must be introduced in a more rigorous way by a coordinate transformation. The resulting symmetric dynamics is then favourably compared with a symmetrized phase portrait reconstructed from the $z$-variable of the Rössler system. Such a link with this latter variable - which is known to be a poor observable of the underlying dynamics - could explain the general difficulty encountered in finding evidence of low-dimensional dynamics in sunspot data.

Key words. chaos - Sun: magnetic fields - Sun: activity - Sun: sunspots

\section{Introduction}

Solar activity is produced by the emergence of magnetic flux through the photosphere forming active regions which include sunspots. The most characteristic feature of solar activity is its basic 11-year cycle discovered by Schwabe (1844). Schwabe also described an irregular behavior with fluctuations in the cycle duration as well as in the individual shape and maximum intensity. The cycles of the sunspot numbers are thus modulated on a time scale longer than the 11-year period. Hale (1919) and co-workers discovered that every 11-years the polarity of the Sun's magnetic field reverses. This feature has been explained for the first time by the dynamo model introduced by Babcock (1961). It is now generally accepted that magnetic cycles in a star like the Sun are produced by a dynamo located at, or near, the base of its convection zone.

Although the global aspects of the solar cycle are well explained by dynamo theory, it remains doubtful whether irregularities are deterministic or stochastic, that is, whether the observations favor an explanation in terms of nonlinear (chaotic) dynamics or stochastic processes. Indeed, none of the studies involving nonlinear dynamical systems theory (Mundt et al. 1991; Kremliovsky 1994; Jinno et al. 1995) provide convincing evidence of a chaotic Sun since no universal method was able to discriminate between colored noise with power law spectra and underlying dynamical processes in data (Theiler et al. 1992). Recent studies have suggested that for an unambiguous detection of low-dimensional deterministic behavior, we will have to wait for the availability of longer and more reliable data sets (Carbonell et al. 1994). Nevertheless, Knobloch and Landsberg (Knobloch et al. 1996, 1998) introduced a six-dimensional model for the magnetic field cycles. Such numerical simulations suggest the presence of low-dimensional chaotic dynamics.

Since the end of the eighties, several different global modeling techniques have been developed for constructing sets of ordinary differential equations or discrete maps (Crutchfield et al. 1987; Giona et al. 1991; Gouesbet 1992; Brown et al. 1994; Letellier et al. 1995). These are particularly powerful techniques for providing a global model from a very limited amount of data. Integrating or iterating these global models may generate synthetic data with the same underlying dynamics. When a satisfactory global model is obtained, clear evidence for a nonlinear deterministic component of the dynamics is thus provided. To the best of our knowledge, a single global model has been presented for the sunspot data (Lainscsek et al. 1998). On the one hand, such a model captures a few characteristics of the sunspot number dynamics, but on the other hand the model is not completely compatible with our understanding of solar dynamics.

Two reasons for the dynamical mismatch may be conjectured. First, it has been noted that the data are not of uniform quality (Eddy 1976). The sunspot number series consists of annual means from 1700 up to 1749 . Between 1749 and 1818 monthly means were used. It was only from 1818 that daily indices were used to construct the time series. Thus, no more than 23 cycles are available. This is definitely not enough for using standard algorithms to search for signatures 
of low-dimensional chaos in time series (like Lyapunov exponents, correlation dimension and so on). Moreover, the sunspot numbers before 1850 were reconstructed by Wolf (Wolf 1952) and are somewhat unreliable since some characteristics of the underlying dynamics are significantly different for the data recorded before and after 1850 (Carbonell et al. 1994). Even after 1850 , the dynamics appears to be non-stationary, that is, there is still some change in the dynamics which cannot be explained in terms of a low-dimensional deterministic system (Carbonell et al. 1994). Since known attempts to construct a global model used the full time series available or the first part of it, this could explain the limited success of previous attempts to obtain global models from the sunspot data, as reported in the literature.

Second, the reversals of the Sun's magnetic field have been introduced using the so-called Bracewell index (Bracewell 1953). Such a procedure presents the disadvantage of forcing the trajectory to pass near the origin of the reconstructed space when switching from one cycle to the next. In that domain, the noise contamination is sufficient to hinder any successful global modeling. An alternative procedure is introduced in this paper.

The paper is organized as follows. In Sect. 2, the problem of reconstructing a phase portrait from the sunspot numbers is addressed and the embedding dimension is estimated. In Sect. 3 it is shown that the phase change can be introduced by a coordinate transformation rather than the Bracewell method. Section 4 is devoted to a comparison between the covers (Letellier \& Gilmore 2001) obtained from the sunspot numbers and those obtained from some variables of the Rössler and the Lorenz systems. Section 5 gives a conclusion.

\section{Phase space reconstruction}

\subsection{The sunspot numbers}

Since we are mainly concerned with the cycle-to-cycle variability, we used the monthly averaged sunspot numbers. We have thus $12 \times 11=131$ data points per cycle which is a reasonable sampling rate for investigating the dynamics using tools borrowed from nonlinear dynamial systems theory. Even when they are monthly averaged, the sunspot numbers still have a considerable level of high-frequency fluctuations (Fig. 1). They therefore need to be smoothed out before any analysis. Many works devoted to the analysis of sunspot number begin by smoothing out the data (Kremliovsky 1994; Lainscsek et al. 1998; Palus \& Novotná 1999; Mininni et al. 2000). Such a smoothing is also used in investigating the dynamics underlying light curves from pulsating stars (Serre et al. 1996; Buchler et al. 2004).

We use a low-pass filter to eliminate the high frequency components. The smoothed data are superimposed on the monthly averaged sunspot number (Fig. 1). The low-pass filter is based on a Fourier transform and a moving window whose size, $w_{\mathrm{s}}$, corresponds to the number of points used. A window size $w_{\mathrm{s}}$ of 30 points - roughly a quarter of an 11-year cycle provides a useful result (Fig. 1). This value will be discussed in the next section.

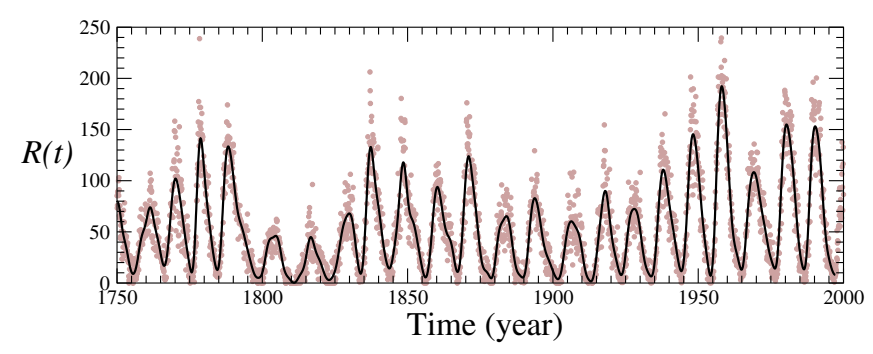

Fig. 1. Monthly averaged sunspot numbers using Wolf's index $R=$ $k(10 g+f)$, where $g$ counts the number of sunspot groups and $f$ counts the individual sun-spots. The factor $k$ was introduced to allow a "normalization" among the different observers who contributed. The time series used here is available on the web-site of the National Geophysical Data Center (NGDC) in Boulder, Colorado, USA at www.ngdc.noaa.gov. The smoothed data $\left(w_{\mathrm{s}}=30\right)-$ thick line are superimposed on the monthly averaged sunspot numbers.

Before any analysis of the dynamics underlying sunspot numbers is carried out, it should be noted that the data are not of uniform quality (Eddy 1976). Indeed, it has been shown that the statistical properties of sunspot numbers differ before and after 1850 (Conway et al. 1998). A simple analysis can be done to verify this. For instance, the variability of the sunspot cycle duration is between 8 and 14 years before 1850 and only between 10 and 12 years after. The transition between the periods of large variability and low variability is around 1850 . Conway and co-workers (Conway et al. 1998) concluded that earlier data should not be used to train neural network that are intended to make predictions at the current epoch.

\subsection{Estimating the embedding dimension}

The first step in investigating the dynamics underlying a scalar time series is to reconstruct a $d$-dimensional phase space (Packard et al. 1980). Two different coordinate sets can be used, namely the delay and the derivative coordinates. Let us start with the delay coordinates. The reconstructed phase space is spanned by

$$
\left\{\begin{array}{l}
u_{1}(t)=R(t) \\
u_{2}(t)=R(t+\tau) \\
u_{3}(t)=R(t+2 \tau) \\
\vdots \\
u_{d}(t)=R(t+(d-1) \tau)
\end{array}\right.
$$

where $\tau$ is the time delay, to be choosen. The estimate of the embedding dimension could be dependent on $\tau$ but we should use a time delay in a range where the estimated dimension does not change with $\tau$. Thus, for estimating the embedding dimension we use the algorithm written by Cao (1997) based on the false nearest neighbor method. The idea is to increase the dimension, $d$, of the phase space up to the point where there are no longer any self-intersections of the trajectory. It is based on the fact that choosing too low an embedding dimension results in points that are far apart in the original phase space being moved closer together in the reconstruction space. But to avoid the choice of a threshold to decide whether a neighbor is false or not, Cao used the relative change in the average distance 


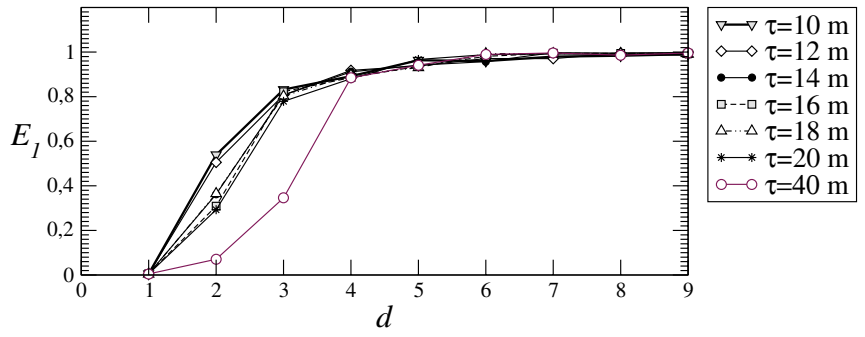

Fig. 2. Relative change in the average distance between neighbor points versus the dimension $d$ of the phase space reconstructed from the smoothed sunspot data. For each value of the time delay $\tau$ (in months), the smoothing parameter $w_{\mathrm{s}}$ is equal to 30 .

between two neighboring points in $\mathbb{R}^{d}$ when the dimension is increased from $d$ to $d+1$. When this index saturates around 1 , the minimum dimension required to embed the trajectory without any self-crossing is reached. This minimal dimension is the so-called embedding dimension $d_{E}$.

We made our computations for different time delays using the 23 available cycles. Indeed, we used the data prior to 1850 since 23 cycles is already under the limit for having a good estimate for the embedding dimension. Of course, the lack of reliability of the earlier data can blurr our results and we have to keep in mind that this is just an estimate. One could object that the saturation value $\left(E_{1} \approx 1\right)$ is not exactly reached for $d=3$ but only for $d$ between 6 and 8 (Fig. 2). However we have to keep in mind that we used the unreliable cycles before 1850 for this estimation and the data, although slightly smoothed, are not noise free. These two reasons can actually affect the dimension estimate by inducing spurious false nearest neighbors.

We illustrate this method by estimating the embedding dimension of the Rössler system (see Sect. 4) using its $x$ variable. This was sampled at a rate equivalent to that used for the monthly sampled sunspot numbers (roughly 120 points per cycle). The phase space is reconstructed with a time delay $\tau=15 \delta t$, i.e. the same value as the one which is retained for most of this analysis. With such a small data set (around 23 cycles), saturation, although better than for the sunspot data, is not observed for $d=3$ (Fig. 3). The saturation is obviously poorer when the data are noise contaminated (with a rate around those of the sunspot data). Note that similar features with other noisy data sets have already been described (Cao 1997). This lack of a complete saturation does not hide the clear change in the slope of the curve $E_{1}(d)$ which is a signature of a space with a sufficiently high dimension. Indeed, the Rössler system can be properly embedded in a 3D space from any of its variables. We therefore estimate that the embedding dimension is three for the smoothed sunspot data as well.

We also computed the embedding dimension for values of the time delay up to 40 months as indicated by an estimation of the best time delay by using the first zero of the auto-correlation function (Liebert \& Schuster 1989). This is one of the possible ways to estimate the time delay (see for instance Kantz (1997) for other techniques). Unfortunately, this value is too large and the phase portrait is too unfolded as shown in Fig. 4. This is also confirmed by the computation of the embedding dimension which clearly shows that a four-dimensional space would

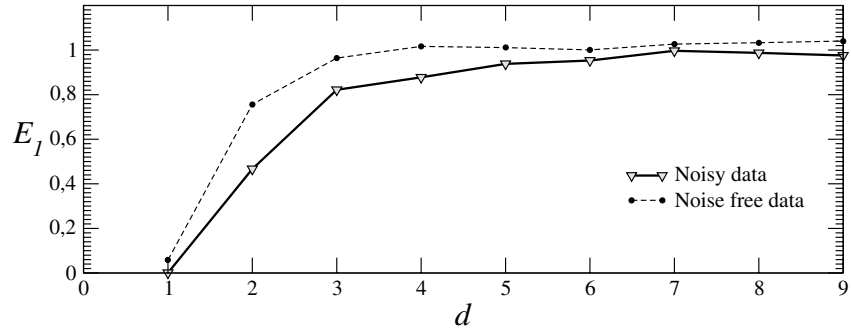

Fig. 3. Relative change in the average distance between neighbor points versus the dimension $d$ of the phase space reconstructed from smoothed noisy data generated by the Rössler system. The time delay is chosen so that there are around 120 points per cycle ( $\tau$ is equal to $15 \delta t$ ). Parameter values for Rössler equations: $a=0.405, b=2$ and $c=4$.

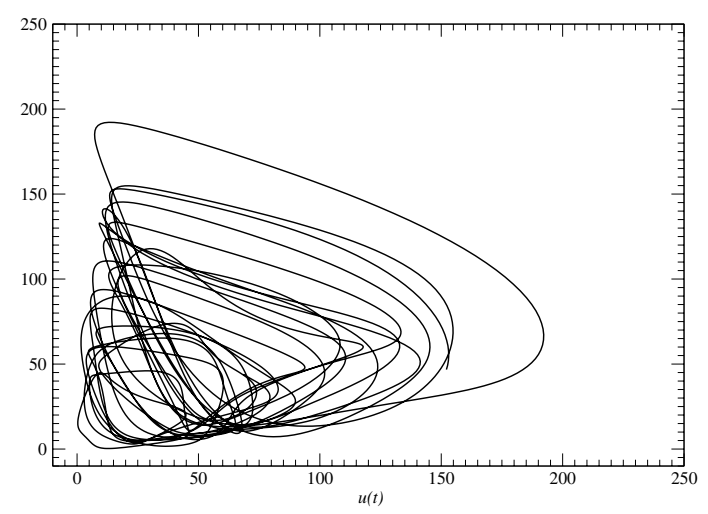

Fig. 4. Phase portrait reconstructed from the sunspot data with a time delay equal to 40 months. It is clearly too unfolded.

be required (Fig. 2) for this time delay. This is a consequence of a spurious structure induced by a too large time delay. It is known that different time delays may lead to different minimum embedding dimensions, especially for time series from continuous time systems (Cao et al. 1998). A good choice of $\tau$ may decrease the embedding dimension which is necessary for phase space reconstruction. In other words, finding a range of time delays for which the minimum embedding dimension does not change constitutes a good indicator that actual properties of the dynamics are identified.

For any time delay $\tau \in[10 ; 20]$, the embedding dimension is not dependent on $\tau$ and is three (Fig. 2). We choose $\tau=16$ months for our analysis, a value which is close to the value used by Mundt et al. (1991). Thus, a three-dimensional phase space should be sufficient to properly unfold the dynamics underlying the sunspot numbers without any self-crossing of the trajectory. Nevertheless, we have to keep in mind that our data set is very short and has very few cycles. Such an estimated embedding dimension must only be considered as an indicator. It suggests that a low-dimensional dynamics could underly the sunspot cycles. Note that an embedding dimension equal to 3 is consistent with the correlation dimensions estimated by Kremliovsky (1994) $\left(D_{2}=2.4 \pm 0.2\right)$ and by Mundt et al. (1991) $\left(D_{2} \approx 2.3\right)$. Although a part of the data is not very reliable, such embedding dimension is a first clue that the dynamics underlying the long-term solar activity might be lowdimensional. 


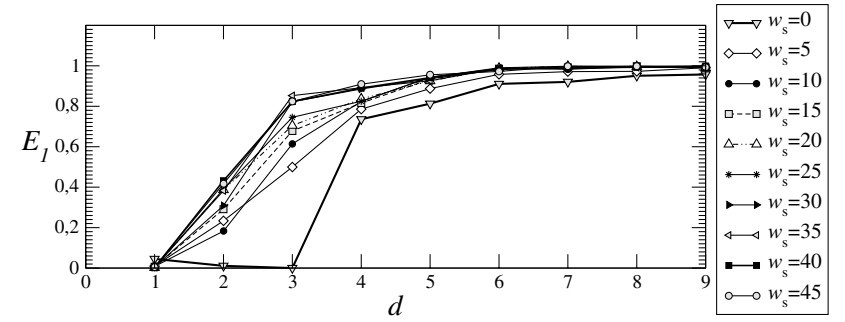

Fig. 5. Relative change in the average distance between neighbor points versus the dimension $d$ of the phase space reconstructed from the smoothed sunspot data. Different values of the smoothing parameter $w_{\mathrm{s}}$ are used. Time delay: $\tau=16$ months.

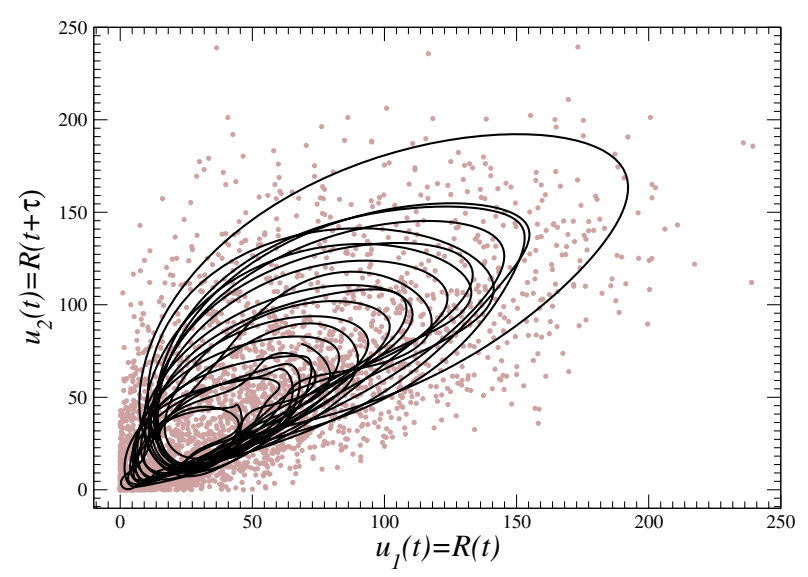

Fig. 6. Plane projection of the phase portrait reconstructed using the delay coordinates $u_{1}=R(t)$ and $u_{2}=R(t+\tau)$ where $\tau=16$ months. Note that the 23 cycles recorded since 1749 are shown here. This explains why some drift may be observed in this phase portrait.

We also checked that the embedding dimension is not excessively dependent on the smoothing parameter $w_{\mathrm{s}}$. Thus we computed the embedding dimension for various values of the smoothing parameter $w_{\mathrm{s}} \in[0 ; 45]$ (Fig. 5). The interesting thing is that the curve tends to be independent on the smoothing parameter when $w_{\mathrm{s}}$ is greater or equal to 30 (we checked that up to $\left.w_{\mathrm{s}} \leq 45\right)$. It therefore seems reasonable to choose the smallest value from this range, that is, $w_{\mathrm{s}}=30$.

A phase portrait is now reconstructed with the estimated parameters. The phase portrait is shown superimposed on the original unsmoothed sunspot numbers (Fig. 6). The phase portrait does not look like a "ball of wool" and there seems to be some structure underlying the data. Obviously, smoothing out the high-frequency fluctuations helps to recover some structure. It is important to note that smoothing can remove part of the original dynamics - in fact, our hope was to remove only the stochastic part - but it cannot inject nonlinear dynamics into the smoothed data.

In order to check that the smoothing proceedure cannot inject deterministic dynamics we randomized the phase of the monthly averaged sunspot number. This is a standard way of producing surrogate data to detect nonlinear determinism in a time series (Theiler et al. 1992). We then applied the same amount of smoothing and obtained the phase portrait shown in Fig. 7. When the surrogate data are smoothed $\left(w_{\mathrm{s}}=30\right)$ and used to reconstruct a phase portrait (Fig. 7), the obtained

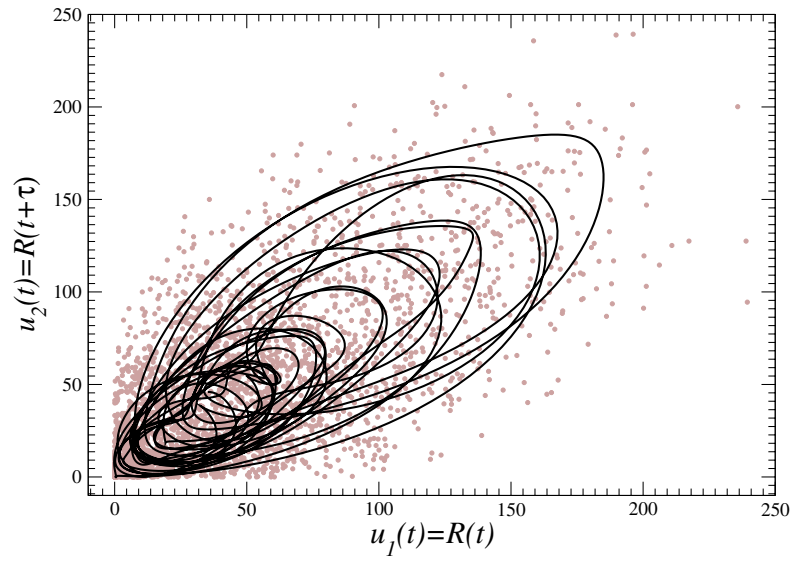

Fig. 7. Phase portrait reconstructed from surrogate sunspot data. The structure is substantially reduced. Reconstruction parameters: $w_{\mathrm{s}}=30$ and $\tau=16$.

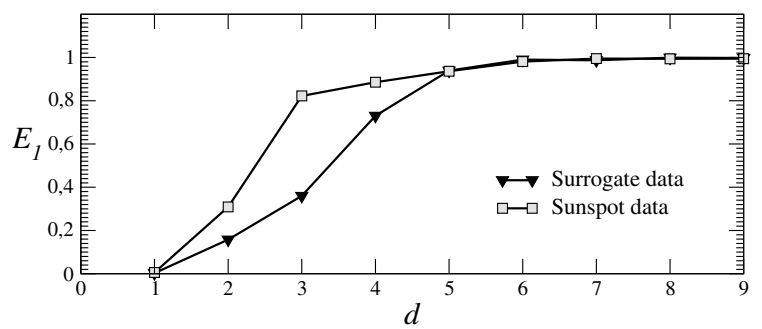

Fig. 8. Embedding dimension estimated from the surrogate data and compared to those estimated from the sunsport numbers. Reconstruction parameters: $\tau=16$ months and $w_{\mathrm{s}}=30$.

dynamics has significant departures from the one induced by the sunspot numbers. There is an obvious lack of regularity in the shape of the cycles and there is no longer a hole in the middle of the attractor, a condition required to properly compute a Poincaré section.

In addition to the previous analysis, we estimate the embedding dimension and compare it to the estimation from the sunspot numbers (with the same reconstruction parameters). It clearly appears that, as expected, the dimension of the phase space reconstructed from the surrogate data is greater than the dimension of the embedding induced by the sunspot number (Fig. 8). Indeed, there is a clear change in the slope of the curve $E_{1}(d)$ for $d=5$. This is another clue to support the hypothesis that the dynamics underlying the smoothed data really correspond to the dynamics underlying the sun activity. We are thus convinced that the dynamics seen in the phase portrait reconstructed from the smoothed data has not been introduced by smoothing but really comes from the original data.

\section{Symmetry properties for the polarity inversion}

Since the 11-year sunspot cycle is driven by the 22-year magnetic field cycle with a polarity inversion every 11 years, it is necessary to introduce some symmetry properties to explicitly show these features. Up-to-now this was done using the socalled Bracewell index (Bracewell 1953) which is defined as the sunspot number with a sign change at the beginning of each period. This index displays a period of 22 years with a 

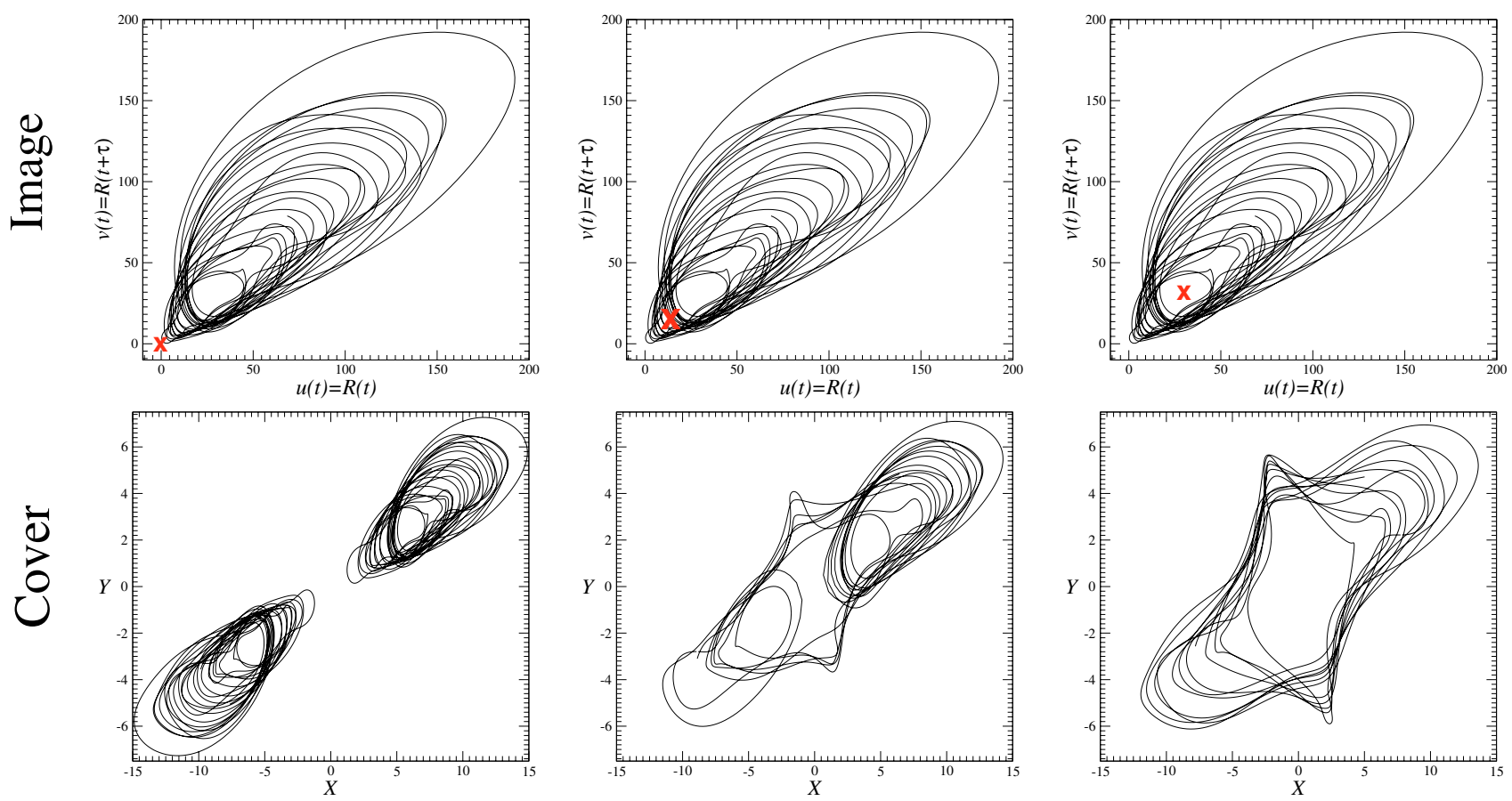

(a) $u_{0}=0$

(b) $u_{0}=15$

(c) $u_{0}=30$

Fig. 9. Different topologically inequivalent covers of the phase portrait reconstructed from the sunspot numbers using the delay coordinates. The location of the singularity - indicated by the cross - is displaced in the plane $\mathbb{R}^{2}(u, v)$ along the bisecting line. Rigorously, case (a) should correspond to the Bracewell index.

sign change every 11 years. This procedure has been used by (Lainscsek et al. 1998; Mininni et al. 2000). Moreover, there is no statistical evidence for a departure between the odd and the even cycles (Conway et al. 1998), thus allowing to think that a symmetry could be involved.

Recently, two of us developed a rigorous way to introduce symmetry properties in systems which have none (Letellier \& Gilmore 2001). Typically, a system without any residual symmetry is called the image system and it is possible to construct a covering dynamical system with a symmetry group $\mathcal{G}$ using a coordinate transformation. The so-called cover thus obtained is locally dynamically equivalent to the image system. The inverse problem is to map a system which is invariant under the symmetry group $\mathcal{G}$ into a locally equivalent dynamical system without any residual symmetry.

In the case of the dynamics underlying the sunspot cycles, the aim is to obtain a dynamics which takes into account the inversion of the magnetic field at every 11-year cycle. This corresponds to an order- 2 symmetry which could be a rotation by $\pi$ around an axis or an inversion. Our procedure accomplishes the original objective for which the Bracewell index (1953) was introduced. The phase portait reconstructed from this time series will be necessarily invariant under an inversion symmetry.

Our aim is to construct a cover of the plane projection spanned by the delay coordinates $\left(u_{1}, u_{2}\right)$ (Fig. 6). The procedure for doing this is straightforward. We map the image coordinates $\left(u_{1}, u_{2}\right)$ to covering coordinates $(X, Y)$ using a simple $2 \leftrightarrow 1$ quadratic mapping given by (Letellier \& Gilmore 2001):

$\Phi=\mid \begin{array}{ll}u_{1}=X^{2}-Y^{2}=\operatorname{Re}(X+i Y)^{2} \\ u_{2}=2 X Y=\operatorname{Im}(X+i Y)^{2}\end{array}$.
This transformation $\Phi: \mathbb{R}^{2}(X, Y) \mapsto \mathbb{R}^{2}\left(u_{1}, u_{2}\right)$ "mods out" the symmetry and has been used for obtaining the image of the Lorenz system, the Burke'n Shaw system, and the Kremliovsky system (Letellier \& Gilmore 2001). In our case, we would like to introduce symmetry. This can be easily done by inverting the map $\Phi$.

When the coordinate transformation $\Phi$ is used, its singularity around which the symmetry is organized is located at the origin of the phase space. This means that we would introduce a symmetry with respect to the origin of the phase space shown in Fig. 6. This is what Bracewell did in introducing a sign change at each minimum of the sunspot cycles. It has been shown by two of us that this is not the only possibility (Letellier \& Gilmore 2001). The singularity can be displaced along the bissecting line of the plane $u_{1}-u_{2}$ by using the map

$\varphi=\mid \begin{aligned} & u_{1} \mapsto u_{1}+u_{0} \\ & u_{2} \mapsto u_{2}+u_{0}\end{aligned}$

where $u_{0}$ defines the position of the singularity in the phase space reconstructed from the sunspot numbers. There are three cases that must be described.

The first case is when the singularity is located at the origin of the phase space $\left(u_{0}=0\right)$. Since it is located outside the attractor, it is not possible to obtain a single connected attractor which is invariant under the symmetry (Letellier \& Gilmore 2001). Two co-existing attractors can only be obtained as shown in Fig. 9a. This means that once the magnetic field has a polarity, it cannot inverse it. Therefore this cannot possibly correspond to what is observed.

The second case occurs when the singularity intersects the attractor. In such a case, the inversion is irregular in time 


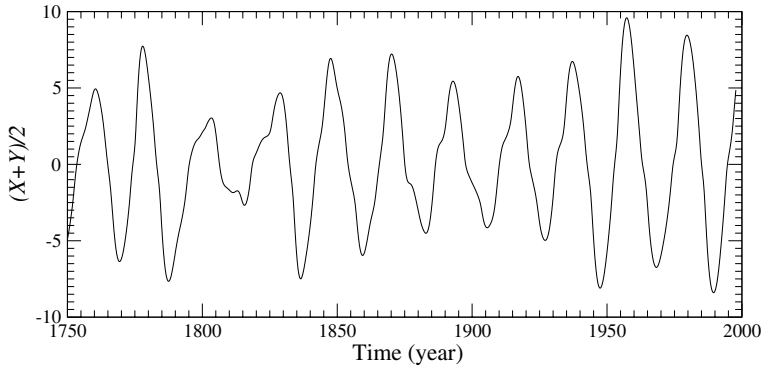

Fig. 10. Time series of the cover of the phase portrait reconstructed from the sunspot numbers.

(Fig. 9b), that is, it does not happen at each cycle, as required. This means that such a cover does not correspond to the dynamics underlying the sunspot cycle either.

The only remaining possibility is to locate the singularity in the hole in the middle of the attractor $\left(u_{0} \in[26 ; 34]\right)$. In this latter case, the cover (Fig. 9c) presents at each cycle an inversion of the polarity of the magnetic field. Increasing $u_{0}$ beyond 34 , induces an intersection between the singularity and the phase portrait and, consequently, irregular inversions of the polarity are again observed. Therefore there is a single possibility for choosing the location of the singularity which matches with the observations, that is when $u_{0}$ is in the range [26;34]. For the remaining part of this work, we will choose to place the singularity at $u_{0}=30$.

The resulting time series has a period of 22 years with an inversion at each 11 year cycle, as required. We chose linear combinations $X \cos (\theta)+Y \sin (\theta)$ of the covering variables $X$ and $Y$ to represent the magnetic field. The angle $\theta$ was varied, and value $\pi / 4$ chosen as the shape of the trace is insensitive to variations about that angle. As a result, we chose the linear combination $\frac{1}{2}(X+Y)$ (Fig. 10) as a representative of the magnetic field. Equal treatment of the cover variables $X$ and $Y$ consistent with the equal treatment of the image variables $u_{1}$ and $u_{2}$ in Eq. (3).

Past attempts to analyse the sunspot dynamics from the Bracewell index could have been biased by the unavoidable discontinuity necessarily introduced by the sign change at the minima, a discontinuity which was removed by using some filters. Here we have proposed a new way to introduce phase changes in the time series.

\section{Equivalence with benchmark systems}

Construction of a cover for sunspot numbers helps not only to introduce in a rigorous way the symmetry properties related to the inversion of the magnetic field polarity, but also to unfold the dynamics near the minima of the 11-year cycles. Moreover, the coordinate transformation $\Phi^{-1}$ provides a twofold cover with a shape that strongly depends on the shape of the "image" phase portrait. The correct application of a proper coordinate transformation can be very useful in revealing subtle differences in the underlying dynamics. Thus, it is a powerful filter to determine whether the dynamics underlying a time series - or equivalently the corresponding reconstructed phase portrait - is close to the dynamics underlying the sunspot numbers. Thus, we will try to identify which dynamical variables from benchmark systems like the Rössler system or the Lorenz system mimic the sunspot cycle.

We start with the three dynamical variables of the Rössler system (Rössler 1976):

$$
\left\{\begin{array}{l}
\dot{x}=-y-z \\
\dot{y}=x+a y \\
\dot{z}=b+z(x-c) .
\end{array}\right.
$$

These equations are integrated using a time step $\delta t$ roughly providing 120 points per cycle to have a resolution similar to that of the sunspot data. From each dynamical variable, we reconstruct a phase portrait using delay coordinates with a time delay $\tau=15 \delta t$ as used for the sunspot cycles. The parameter values are chosen to have a hole in the middle of the attractor with a reasonable size. Then, we apply a rigid displacement of the attractor to place the singularity of the transformation at the center of this hole, as done in Fig. 9c for the sunspot numbers. Each reconstructed phase portrait - which plays the role of the image system - and the corresponding two-fold cover obtained using the inverted map $\Phi^{-1}-$ are shown in Fig. 11.

The three reconstructed phase portraits - the three images shown in the first row of Fig. 11 - may be split in two groups. First, the phase portraits induced by the $x$ and the $y$ variables have a very different shape than the portrait induced by the sunspot cycles (Fig. 6). In particular, the hole is more or less at the center of the phase portrait, a characteristic which is not present in the sunspot phase portrait. The two-fold covers (Figs. 11a and 11b) present different shapes when compared to the cover of the sunspot cycles (Fig. 9c). Therefore variables $x$ and the $y$ of the Rössler system are dynamically quite different from the sunspot number.

The phase portrait reconstructed from the $z$-variable is clearly the most similar to the portrait induced by the sunspot cycles. The hole is located near the origin (Fig. 11c, top) as is the case for the portrait induced by the sunspot numbers. The folding is not clearly seen, since it occurs in the small neighborhood of the minima. When the cover is created, a symmetric phase portrait very similar to the cover of the sunspot cyles is obtained (Fig. 11c, bottom). This provides strong evidence that the underlying dynamics of the sunspot cycles may be similar to the Rössler system, particularly as expressed by its $z$ variable. This suggests that the dynamo dynamics could have a low degree of observability, as does Rössler system observed from the $z$-variable (Letellier \& Aguirre 2002). This could account, in part, for the difficulties encountered by many researchers in analysing and modeling sunspot dynamics.

The comparison is even better when the square root of the $z$-variable is used (Fig. 12). In order to improve the "simulation", we integrated the Rössler system with a multiplicative noise and smoothed out the $\sqrt{z}$-variable. The smoothing parameter is the same as the one used for smoothing the sunspot numbers. A cover derived from this smoothed noisy time series is shown in Fig. 12c. This noisy cover has a very similar shape to the cover directly obtained from the smoothed sunspot cycles (Fig. 9c). 

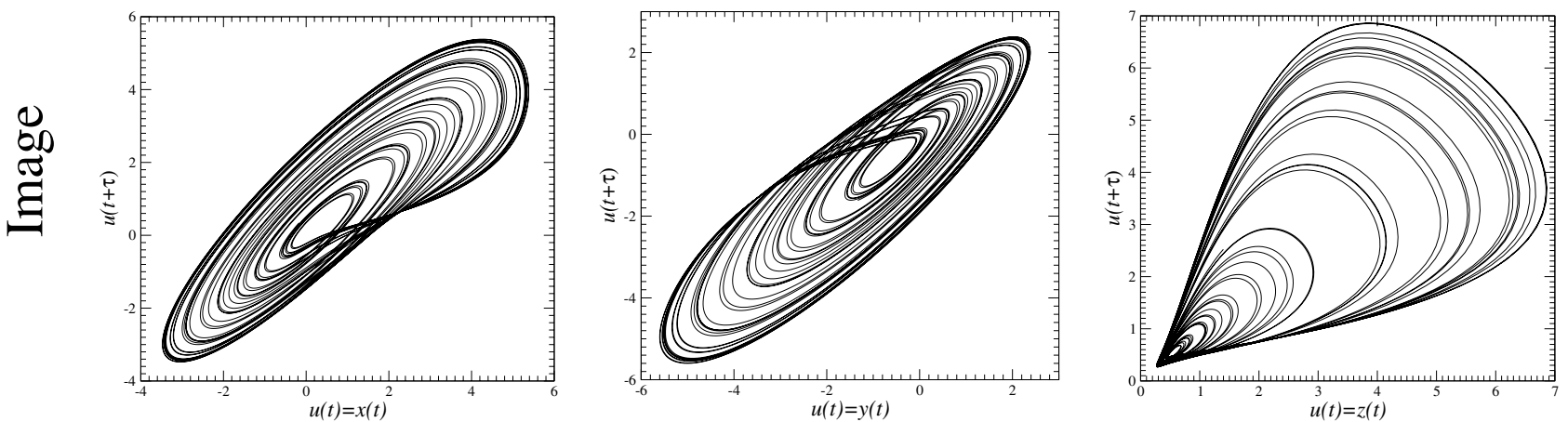

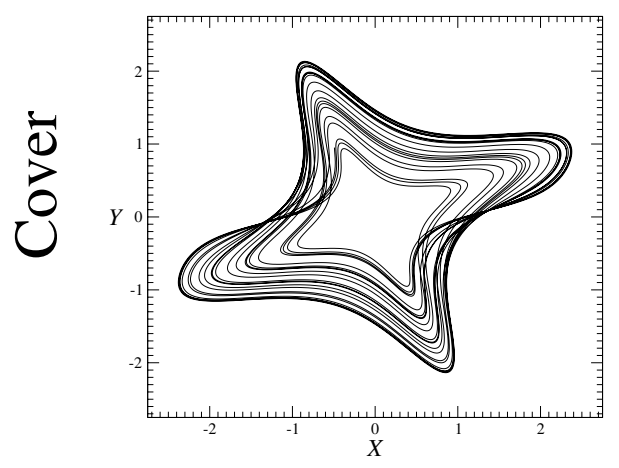

(a) $x$-variable $u_{0}=0.4$

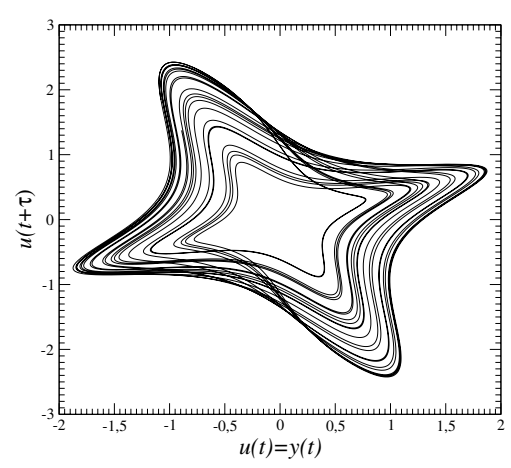

(b) $y$-variable $u_{0}=-0.6$

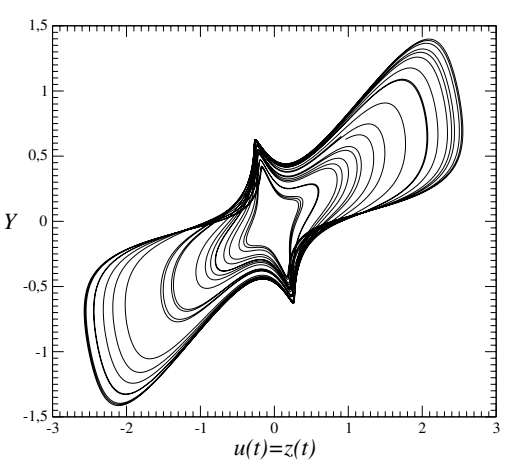

(c) $z$-variable $u_{0}=0.6$

Fig. 11. Different covers of the chaotic attractor reconstructed from the dynamical variables of the Rössler system. Parameter values: $a=0.42$, $b=2.0$ and $c=4.0$.

We also compared the cover of the sunspot cycles with the cover obtained from the phase portrait induced by the $z$-variable of the Lorenz system (Lorenz 1963):

$\left\{\begin{array}{l}\dot{x}=\sigma(y-x) \\ \dot{y}=R x-y-x z \\ \dot{z}=-b z+x y\end{array}\right.$

where the parameters have the usual values. The cover obtained (Fig. 13) is obviously very different from the sunspot cover and, consequently, the Lorenz dynamics seen from the $z$-variable and dynamics underlying the sunspot cycles are very different. A comparison with the phase portrait reconstructed from the $x$ or $y$ variable is not necessary because, in the case of the Lorenz system, these variables are mapped to their opposites under the rotation symmetry around the $z$-axis. This means that these two variables already provide covers of the image of the Lorenz system (Letellier \& Gilmore 2001). Moreover, as it is well known, the trajectory switches from one "wing" to the other in an irregular manner. These covers - or equivalently, the phase portrait reconstructed from the $x$ or the $y$ variable thus correspond to the cover obtained from the sunspot cycles when the singularity intersects the image attractor as shown in Fig. 9b. In other words, it corresponds to an irregular inversion of the magnetic field polarity. These two variables cannot reproduce the most relevant characteristics of sunspot dynamics. The Lorenz system is therefore not a good model for the dynamics underlying the sunspot cycles.

To end these comparisons, we built two-fold covers from the phase portrait induced by the surrogate data computed from the sunspot cycles (Fig. 7). The cover obtained (Fig. 14) has no longer the regularity observed in the sunspot data. Only the rough shape is preserved. In particular, there are some small "loops" within each wing which do not occur in the observational data. Thus, we conclude that the dynamics underlying the sunspot numbers is very similar to those of the Rössler system investigated from its $z$-variable - or more accurately, the $\sqrt{z}$-variable. This is rather strong evidence that the mechanism driving sunspot dynamics exhibits low dimensional chaotic behavior. We note that a link with the Rössler dynamics was already discussed by Kremliovsky (1994).

Since we established that the sunspot time series is rather similar to the $z$ time series of the Rössler system, a few comments will be given about the specificity of this variable. Although coming from a quite simple system - the Rössler equations - this variable is known for providing a very poor observability of the Rössler dynamics (Letellier et al. 1998, 2002). In practice, this means that measuring the $z$-time series does not allow us to recover the whole dynamics. In particular, many attempts for getting a global model from the noise free $z$-variable failed. Only a specific structure selection (Lainscsek et al. 2003) or increasing the embedding dimension (Letellier et al. 1998) allowed to succeed in such a task. This, in addition to the short record and some nonstationarity, would explain why no successful autonomous global models were obtained from the sunspot numbers.

\section{Conclusion}

Sunspot numbers have been investigated using some tools borrowed from nonlinear dynamical systems theory. It has been shown that, when the long term dynamics is investigated, an embedding dimension equal to three is be sufficient. By using 


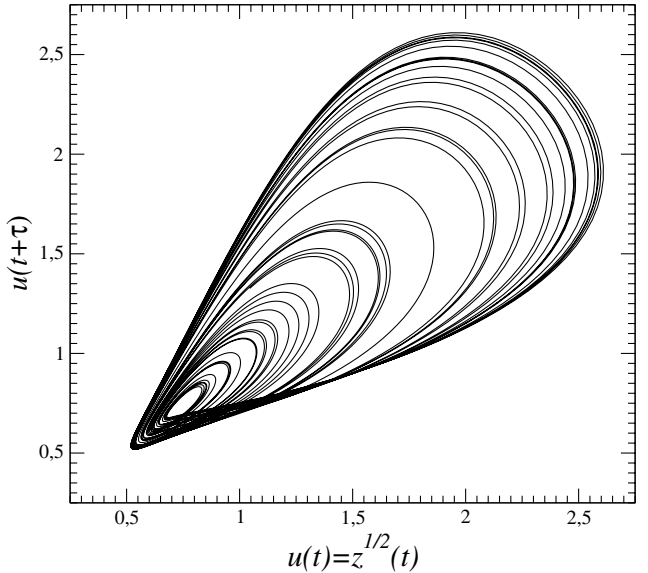

(a) Rössler system

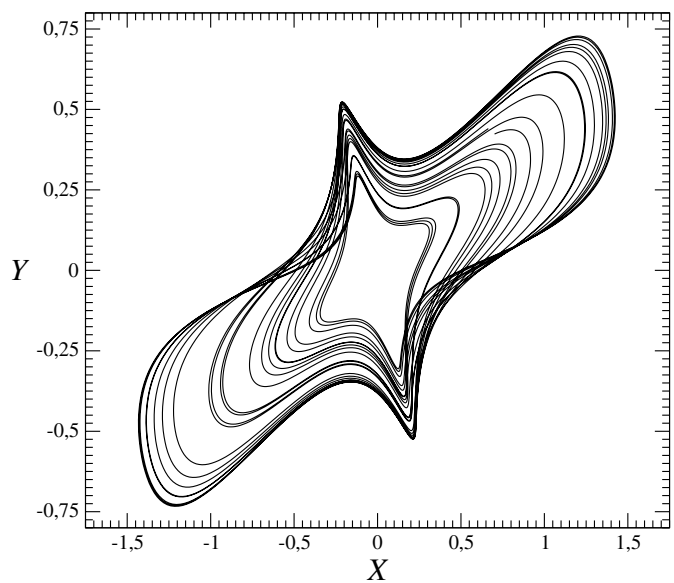

(b) 2-fold cover of the noise free Rössler dynamics

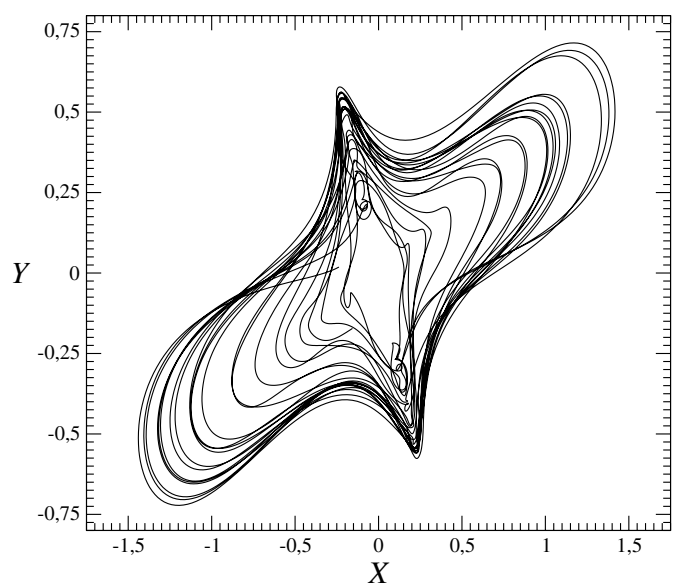

(c) 2-fold cover of the smoothed noisy Rössler dynamics

Fig. 12. Two-fold cover of the phase portrait reconstructed from the $\sqrt{z}$-variable of the Rössler system. Parameter values: $a=0.42, b=2.0$ and $c=4.0$.

the recent theory developed for covering dynamical systems, it was shown that it is more useful to introduce a symmetry by introducing a $2 \rightarrow 1$ coordinate transformation rather than changing sign by hand using the Bracewell index. Indeed, the dynamics that follow introduction of the Bracewell index is not natural because some discontinuities are introduced "by hand"

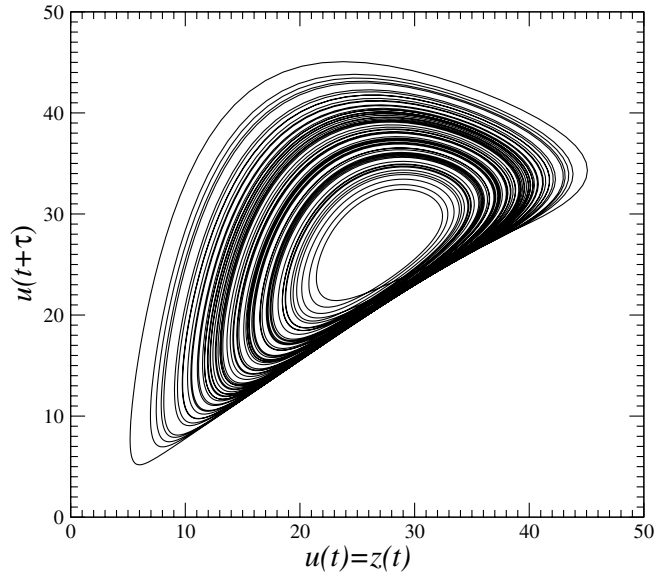

(a) Lorenz chaotic attractor

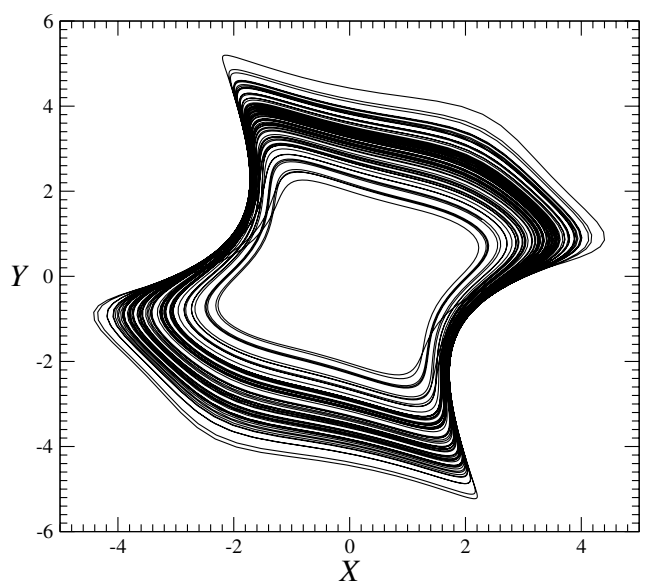

(b) Two-fold cover

Fig. 13. Two-fold cover of the phase portrait reconstructed from the $z$-variable of the Lorenz system. Parameter values: $R=28, \sigma=10$ and $b=8 / 3$.

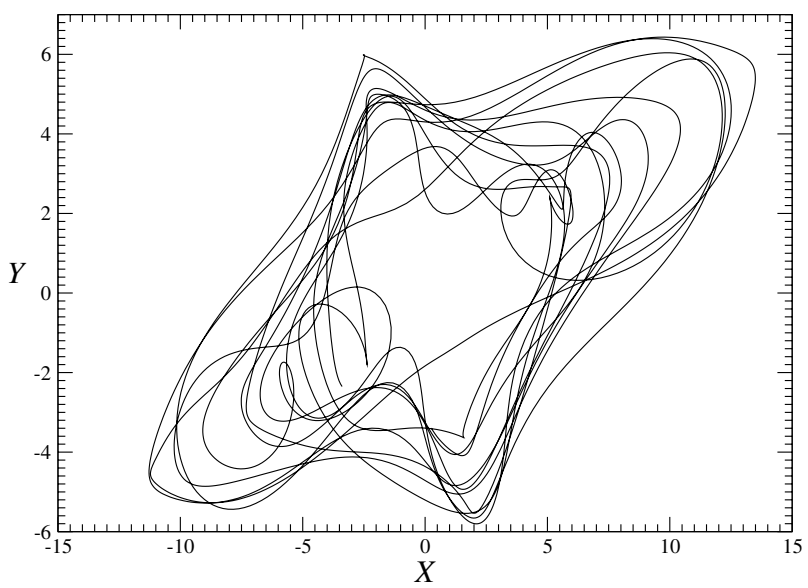

Fig. 14. Two-fold cover of the phase portrait reconstructed from the surrogate data computed from the sunspot cycles. The cover does not present the 11-year alternation observed in the dynamics underlying the sunspot cycles.

with this index and subsequently filtered away. This could explain why previous searches for low-dimensional chaotic 
dynamics have been unsuccessful. We therefore propose a new way to introduce the phase change after each sunspot cycle.

We then compared the so-obtained cover of the sunspot number with the cover of the phase portrait reconstructed from the three variables of the Rössler system and the $z$-variable of the Lorenz system. The $z$-variable of the Rössler system is clearly the best "simulation" for the sunspot cycles. We noted that using $\sqrt{z}$ slightly improves the resemblance with sunspot cycles. Unfortunately, the $z$-variable of the Rössler system is recognized as being a poor observable of the underlying dynamics, a fact that could explain why so many works lead to a lack of evidence for a low-dimensional dynamics underlying the sunspot cycles. We believe that using the appropriate cover of the sunspot cycles could help to unfold the dynamics and provide a better observability of the dynamics.

Acknowledgements. Part of this work was supported by CNRS and CNPq.

\section{References}

Aguirre, L. A., \& Letellier, C. 2005, J. Phys. A, 38, (28), 6311

Babcock, H. W. 1961, Astr. J., 133, 572

Bracewell, R. N. 1953, Nature, 171, 649

Brown, R., Rul'kov, N. F., \& Tracy, E. R. 1994, Phys. Lett. A, 194, 71

Buchler, J. R., Kolláth, Z., \& Cadmus, R. R. 2004, Astr. J., 613, 532

Cao, L. 1997, Physica D, 110, 43

Cao, L., Mees, A., \& Judd, K. 1998, Physica D, 121, 75

Carbonell, M., Oliver, R., \& Ballester, J. L. 1994, A\&A, 290, 983

Conway, A. J., Macpherson, K. P., Blacklaw, G., \& Brown, J. C. 1998, J. Geophys. Res. A, 103, 29733

Crutchfield, J. P., \& McNamara, B. S. 1987, Complex Sys., 1, 417

Eddy, J. A. 1976, Science, 192, 1189

Giona, M., Lentini, F., \& Cimagalli, V. 1991, Phys. Rev. A, 44, 3496
Gouesbet, G. 1992, Phys. Rev. A, 46, 1784

Hale, G. E., Ellerman, F., Nicholson, S. B., \& Joy, A. H. 1919, Astr. J., 49, 153

Jinno, K., Xu, S., Berndtssen, R., Kawamura, A., \& Matsumoto, M. 1995, J. Geophys. Res. A, 100, 14773

Kantz, H., \& Schreiber, T. 1997, Nonlinear time series analysis (Cambridge University Press), 1997

Knobloch, E., \& Landsberg, A. S. 1996, MNRAS, 278, 294

Knobloch, E., Tobias, S. M., \& Weiss, N. O. 1998, MNRAS, 297, 1123

Kremliovsly, M. 1994, Sol. Phys., 151, 351

Lainscsek, C., Schürrer, F., \& Kadtke, J. B. 1998, Int. J. Bif. Chaos, 8, 899

Lainscsek, C., Letellier, C., \& Gorodnitsky, I. 2003, Phys. Lett. A, 314,409

Letellier, C., \& Gilmore, R. 2001, Phys. Rev. E, 63, 16206

Letellier, C., \& Aguirre, L. A. 2002, Chaos, 12, 549

Letellier, C., Le Sceller, L., Dutertre, P., et al. 1995, J. Phys. Chem., 99, 7016

Letellier, C., Maquet, J., Le Sceller, L., Gouesbet, G., \& Aguirre, L. A. 1998, J. Phys. A, 31, 7913

Liebert, W., \& Schuster, H. G. 1989, Phys. Lett. A, 142, 107

Lorenz, E. N. 1963, J. Atm. Sci., 20, 130

Mininni, P. D., Gomez, D. O., \& Mindlin, G. B. 2000, Phys. Rev. Lett., 85,5476

Mundt, M. D., Maguirre, W. B. II, \& Chase, R. R. P. 1991, J. Geophys. Res. A, 96, 1705

Packard, N. H., Crutchfield, J. P., Farmer, J. D., \& Shaw, R. S. 1980, Phys. Rev. Lett., 45, 712

Palũs, M., \& Novotná, D. 1999, Phys. Rev. Lett., 83, 3406

Rössler, O. E. 1976, Phys. Lett. A, 57, 397

Schwabe, H. 1844, Astr. Nach., 21, 254

Serre, T., Kolláth Z., \& Buchler, J. R. 1996, A\&A, 311, 833

Theiler, J., Eubank, S., Longtin, A., Galdrakin, B., \& Farmer, J. D. 1992, Physica D, 58, 77

Wolf, R. 1852, CRAS, 35, 704 